\title{
Technology Rhinko. The History and Present State.
}

\author{
Vladimir Kozlov, Stepan Kudryashov.
}

\author{
ENT Department Central State Medical Academy of the Department \\ OF Presidential Affairs, Moscow, Russian Federation.
}

Paranasal sinusitis (PNS) in the past and present, and well into the future is one of the serious problems in rhinology. Methods of treatment of PNS are divided into conservative and surgical. The widespread use in practice of functional endoscopic sinus surgery (FESS) showed not only advantages, but also revealed notable shortcomings of this method. According to all clinical recommendations, systemic antibiotic therapy remains the main method of treatment for PNS. Given the increase in resistance to antibiotics, this cannot be considered justified. Development of methods of PNS treatment based on local influence in the focal point of inflammation may become a priority research area.

The first publication on the effectiveness of treatment of PNS by creating negative pressure in the nasal cavity was made by P. Gellat in 1911. In 1926, A. Proetz published a method for moving fluids for the purpose of PNS treatment. Both of these methods are based on changing the pressure in the nasal cavity and paranasal sinuses. At the same time, in both methods pressure cannot be controlled. In the period from 1981 to 1992 we developed a method of treatment of PNS, which was based on controlled pressure in the nasal cavity and paranasal sinuses. In the same time frame, five catheter models were developed for which patents of the invention were obtained. To name this method, the acronym "YAMIK" was proposed, which meant "Yaroslavl, Markov and Kozlov". Unfortunately, at present we do not have the right to use this acronym, as in 1991 (unbeknownst to us) it was stolen from us and registered as a trademark.

The first model of the catheter was a three-channel tube on which two balloons were rigidly fixed without the possibility of changing the distance between them. A "working" channel was opening in the space between the balloons (Patent SU1311714A1). The disadvantage of the catheter was that an individual catheter was required for each nose with a specific distance between the balloons. In the second model of the catheter, one of the balloons was made movable which allowed the use of the same catheter for people with different length of the nasal cavity (Patent SU1715327A1). However, the mobility of the balloon was limited, which required to have several types of catheter. In the third model the problem of choosing the distance between the balloons was solved by means of the fact that the tube with the "working" channel was made inside the movable cuff (Patent SU1768141A1). This model was first introduced in Japan at the ISIAN congress in 1991.

At this congress, Prof. Tadami Kumazawa showed interest in our invention. In 1992, Prof. Kumazawa came to Russia together with Dr. Kubo and Dr. Ino. The first international conference dedicated to this method was held in Yaroslavl. Subsequently, in 1993 Prof. Kumazawa sent to us Dr. Nakamura, who within a month fully mastered the method. During one of my visits to Japan, Prof. Kumazawa introduced me to Prof. Goro Mogi, Prof. Masaru Ohyama, Prof. Hiroshi Moriyama, Prof. Hideyuki Kawauchi, Prof. Yuichi Kurono, Prof. Shoji Matsune. In the course of several years we have conducted several training courses for doctors in Tokyo, Kagoshima, Oita, Sendai, Izumo.

The culmination of these visits was the receipt in 1998 of the permission of the Ministry of Health in Japan to use the catheter in clinical practice and its inclusion in the compulsory insurance system. However, the method was designated as a method of washing the nasal cavity, and not as a method of treatment. As a result, it was not beneficial for doctors to use it in practice, whereas the company stopped supplying catheters to Japan.

In 2016, at the ERS congress in Stockholm, Prof. 
Kawauchi introduced us to Dr. Tsukada. Dr. Tsukada said that in 2014, Japanese colleagues asked him to please start manufacturing catheters, as this method of treatment helps many patients, whereas the Russian company does not supply them. Dr. Tsukada's company has designed a new catheter model called "ENT-DIB." Indisputably, the key positive point of this model was its manufacture from silicone. The company's engineers introduced a new element into the catheter design, creating an additional channel with a diameter of about $1 \mathrm{~mm}$ inside the tube for inflating the nasopharyngeal balloon, which, in their opinion, provided for nasal breathing during the procedure. We really liked the ENT-DIB catheter, especially the quality of implementation and the material from which it was made. Dr. Tsukada presented us with several catheters. Upon returning home, we put them into practice. In process of application it was found that the patient during the procedure cannot breathe through the additional channel, as when the balloon inflates, the channel opening comes in contact with the back of the nasopharynx and is blocked by it. Unfortunately, the ENTDIB catheter also inherited the drawbacks of the YAMIK catheter. Experimentally, we found that when negative pressure is created in the nasal cavity, the inferior turbinate sharply increases in size (due to the blood supply of thinwalled venous sinusoids), almost reaching the nasal septum and therefore filling the general nasal passage (meatus nasi communis). That is why for some patients, when negative pressure is created, the opening of the "working" channel gets closed by the mucous membrane of the inferior turbinate. The blockade of the opening of the "working" channel does not allow to evacuate the secretion from the paranasal sinuses. To solve this problem, we made design changes to this catheter. In the new model of the catheter, the "working" channel passes in a tube that is made movable relative to the cuff. This design solution allows not only to change the distance between the balloons, but also to bring the tube with the "working" channel directly to the middle nasal meatus. As a result, the opening of the "working" channel cannot be blocked by the inferior turbinate. The new catheter is made of silicone. A patent for an invention was obtained for this catheter (Patent 2663932 dated 13.08.2018; International application No. PCT/RU2018/000416, dated 22.06.2018).

We have conducted studies of a new catheter, which confirmed the possibility of injecting the radiopaque solution into the paranasal sinuses, as well as the possibility of evacuating the pathological secretion from them in acute rhinosinusitis.

Considering the fact that at present we cannot use the acronym "YAMIK", we propose a new name for the method: Technology “RHINKO”. 